\title{
Events during the 3 Months Immediately Preceding Onset of Chronic Urticaria: A Questionnaire Study
}

\author{
Sedra Ali ${ }^{\mathrm{a}}$ Misbah Noshela Ghazanfar ${ }^{\mathrm{a}}$ Jesper Grønlund Holm ${ }^{\mathrm{a}}$ \\ Simon Francis Thomsen ${ }^{a, b}$ \\ ${ }^{a}$ Department of Dermatology, Bispebjerg Hospital, Copenhagen, Denmark; ${ }^{b}$ Department of Biomedical Sciences, \\ University of Copenhagen, Copenhagen, Denmark
}

Chronic urticaria $(\mathrm{CU})$ is an itching skin disease characterized by wheals and/or angioedema for more than 6 weeks. CU can be divided into chronic spontaneous urticaria (CSU), which is the most common subtype, and chronic inducible urticaria (CINDU). The triggers that lead to the urticarial signs and symptoms in CINDU patients are mainly physical or chemical stimuli. Physical stimuli are pressure (in delayed pressure urticaria), radiation (in solar urticaria), friction (in symptomatic dermographism), temperature (in cold and heat urticaria) and vibration (in vibratory angioedema). Chemical triggers of CINDU reactions are water (in aquagenic urticaria), sweat (in cholinergic urticaria) and other urticariogenic chemical compounds (in contact urticaria) $[1,2]$. The course of symptoms in CU is highly unpredictable and symptom control can be challenging despite availability of effective treatment options for antihistaminerefractory patients.

The pathogenesis of $\mathrm{CU}$ is thought to be auto-immune and/or auto-allergic with circulating IgG and IgE autoantibodies, respectively, directed against soluble and tissue antigens [3]. Although these factors can be demonstrated in a sizable proportion of patients, it is not known whether they occur before or after the first onset of symp- toms and to which extent external eliciting factors play a role in disease onset and antibody formation. Identification of such external eliciting factors for $\mathrm{CU}$ can guide preventive strategies. This study aimed to determine the frequency of everyday life events that occurred during the 3 months immediately preceding onset of CU.

A questionnaire on various everyday life events was developed inspired by The Holmes-Rahe Stress Inventory [4] and pilot-tested among a small group of patients with $\mathrm{CU}$ to allow feedback and subsequent modification. Thereafter, the questionnaire was administered consecutively to all newly referred patients with onset of CU within the past 3 years from a tertiary dermatological referral centre for CU. Data on disease characteristics, blood samples and patient-reported outcomes (Urticaria Activity Score in the past 7 days [UAS7] and Dermatology Life Quality Index [DLQI]) were obtained by clinical examination and interview.

A total of 85 patients with a mean age of 37.8 years were included. CSU was present in $70.6 \%$, whereas $10.6 \%$ had only CINDU and $18.8 \%$ had CSU with concomitant CINDU. Angioedema was present in $41.2 \%$, while $14.1 \%$ had a positive urticaria histamine release (HR) test. The mean UAS7 score was 23.5 points and the mean DLQI score was karger@karger.com

(c) 2020 S. Karger AG, Basel

www.karger.com/drm

Karger!
Simon Francis Thomsen

Department of Dermatology, Bispebjerg Hospital

Bispebjerg Bakke 23

DK-2400 Copenhagen NV (Denmark)

E-Mail simonfrancisthomsen@gmail.com 
Table 1. Characteristics of the included patients and frequency of everyday life events immediately preceding the onset of chronic urticaria $(n=85)$

\begin{tabular}{lr} 
Clinical characteristics & \\
Female sex, $n$ (\%) & $67(78.8)$ \\
Age, years, mean (SD) & $37.8(16.4)$ \\
Smoking, $n$ (\%) & $17(20.0)$ \\
First-degree relative with CU, $n(\%)$ & $14(16.5)$ \\
CSU, $n$ (\%) & $60(70.6)$ \\
CINDU, $n(\%)$ & $9(10.6)$ \\
CSU + CINDU, $n$ (\%) & $16(18.8)$ \\
Age of onset of CU, years, mean (SD) & $36.0(17.0)$ \\
Duration of CU, years, mean (SD) & $1.7(2.3)$ \\
Angioedema, $n(\%)$ & $35(41.2)$ \\
Positive urticaria histamine release test, $n(\%)$ & $12(14.1)$ \\
Serum total IgE, KIU/L, median (range) & $52(1-782)$ \\
Thyroid disease & $15(17.6)$ \\
Anxiety/depression & $8(9.4)$ \\
Asthma & $16(18.8)$ \\
Hay fever & $21(24.7)$ \\
UAS7, mean (SD) & $23.5(14.2)$ \\
DLQI, mean (SD) & $9.7(7.2)$ \\
\hline Everyday life events that occurred immediately & \\
before onset of CU, $n$ (\%) & \\
Experienced emotional stress & $37(43.5)$ \\
Travelling abroad & $17(20.0)$ \\
Had an infection & $16(18.8)$ \\
Had a new job & $14(16.5)$ \\
Weight changes & $12(14.1)$ \\
Experienced unusual physical symptoms & $9(10.6)$ \\
Moved house & $9(10.6)$ \\
Initiated new medication(s) & $7(8.2)$ \\
Changes in physical activity habits & $7(8.2)$ \\
Major dietary changes & $6(7.1)$ \\
Eaten new foodstuff & $3(3.5)$ \\
Changes in smoking habits & $3(3.5)$ \\
Changes in alcohol drinking habits & $3(3.5)$ \\
Onset of another chronic illness & $3(3.5)$ \\
Been pregnant or tried to become pregnant & $2(2.4)$ \\
\hline
\end{tabular}

CU, chronic urticaria; CSU, chronic spontaneous urticaria; CINDU, chronic inducible urticaria; UAS7, Urticaria Activity Score in the past 7 days; DLQI, Dermatology Life Quality Index; $\mathrm{SD}$, standard deviation.

9.7 points. Having experienced emotional stress (43.5\%), travelled abroad (20.0\%), had an infection (18.8\%), had a new job (16.5\%) and weight changes (14.1\%) were the most frequent life events reported to have occurred in the 3 months immediately preceding onset of CU (Table 1).

Patients who had experienced emotional stress prior to $\mathrm{CU}$ debut more often developed angioedema (59.5 vs. $27.1 \%, p=0.003)$ and tended more often to have CINDU ( 37.8 vs. $22.9 \%, p=0.134$ ), to have a positive urticaria HR test ( 21.6 vs. $8.3 \%, p=0.081)$, to be of younger age ( 35.1 vs. 39.7 years, $p=0.207)$ and to have higher UAS7 (25.8 vs. $21.7, p=0.194)$ and DLQI scores (11.2 vs. $8.5, p=$ $0.087)$. Also, patients reporting a recent infection tended to more often have a positive urticaria HR test ( $25.0 \mathrm{vs.}$ $11.9 \%, p=0.233$ ), be of younger age ( 35.1 vs. 39.7 years, $p=0.207)$ with higher UAS7 (25.8 vs. $21.7, p=0.194)$ and DLQI scores (11.2 vs. 8.5, $p=0.087$ ).

External eliciting factors, as those explored in our questionnaire, have previously been suggested to be implicated in the pathogenesis of CU but have not formally been examined in relation to disease onset [1]. Emotional stress and having had an infection were among the most frequently reported everyday life events to have occurred immediately before onset of CU symptoms, and these factors were associated with a higher occurrence of angioedema, CINDU, autoimmunity, more severe urticaria symptoms and lower quality of life. However, results were only statistically significant regarding the relationship between emotional stress and subsequent angioedema. Determination of causality of such factors, however, is difficult due to the complexity of $\mathrm{CU}$ pathogenesis and because a suspected eliciting factor may be a cause, an aggravating factor or unrelated to CU. This is supported by previous findings that emotional stress serves as an exacerbating factor of CU symptoms [5]. The mechanisms whereby several of these external factors may possibly act in concert with immunogenetic factors to induce auto-antibody formation and symptoms of CU are elusive. Unfortunately, this study cannot be used to infer causality mainly because of the retrospective design and because comparisons with healthy control subjects or patients with other acute or chronic skin diseases were not performed. Therefore, the frequency of these factors in a non-CU control population is not known. Moreover, the relationship between events that occurred up to 3 years before the determination of disease activity, quality of life and blood biomarkers also makes causal inference difficult. Still, the fact that certain relationships were suggested in this study signals that a larger prospective study with more precise determination of life events could be of value. Consequently, this study should encourage further exploration of mind-body interaction [6], stressful life events, infections and other external factors in the aetiology of CU.

\section{Key Message}

Patients with chronic urticaria frequently report emotional stress, travelling abroad, an infection, job change and weight changes to have occurred immediately before disease onset. 


\section{Statement of Ethics}

This research was conducted ethically in accordance with the World Medical Association Declaration of Helsinki. Since only questionnaire data and routinely sampled clinical data were used, informed consent was not needed as per Danish law.

\section{Disclosure Statement}

The authors have no conflicts of interest to declare.

\section{Funding Sources}

The study received no funding.

\section{Author Contributions}

Sedra Ali performed the analyses and wrote the first draft of the manuscript, Misbah Noshela Ghazanfar and Jesper Grønlund Holm developed the questionnaire and collected the data. Simon Francis Thomsen conceived the idea, performed the analyses and collected the data. All authors critically revised and approved the final version of the manuscript.

\section{References}

1 Zuberbier T, Aberer W, Asero R, Abdul Latiff $\mathrm{AH}$, Baker D, Ballmer-Weber B, et al. The EAACI/GA ${ }^{2} \mathrm{LEN} / \mathrm{EDF} / \mathrm{WAO}$ guideline for the definition, classification, diagnosis and management of urticaria. Allergy. 2018 Jul; 73(7):1393-414.

2 Maurer M, Hawro T, Krause K, Magerl M, Metz M, Siebenhaar F, et al. Diagnosis and treatment of chronic inducible urticaria. $\mathrm{Al}$ lergy. 2019 Dec;74(12):2550-3.

3 Bracken SJ, Abraham S, MacLeod AS. Autoimmune theories of chronic spontaneous urticaria. Front Immunol. 2019 Mar;10: 627.

4 Noone PA. The Holmes-Rahe Stress Inventory. Occup Med (Lond). 2017 Oct;67(7):581-2.

5 Maurer M, Weller K, Bindslev-Jensen C, Giménez-Arnau A, Bousquet PJ, Bousquet J, et al. Unmet clinical needs in chronic spontaneous urticaria. A GA ${ }^{2} \mathrm{LEN}$ task force report. Allergy. 2011 Mar;66(3):317-30.

6 Broom BC. A reappraisal of the role of 'mindbody' factors in chronic urticaria. Postgrad Med J. 2010 Jun;86(1016):365-70. 